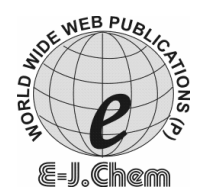

http://www.e-journals.net
ISSN: 0973-4945; CODEN ECJHAO

E-Journal of Chemistry

2009, 6(2), 348-356

\title{
Synthesis and Biological Activity of Some 3,5-Diaryl- 1-benzothiazolopyrazoline Derivatives: Reaction of Chalcones with 2-Hyrazinobenzothiazoles
}

\author{
VANDANA SHARMA* and K. V. SHARMA \\ Department of Engineering Chemistry, \\ Mahakal Institute of Technology, Ujjain -456664, India. \\ vandanak_sharma@yahoo.co.in
}

Received 21 July 2008; Accepted 10 September 2008

\begin{abstract}
A series of 3,5-diaryl-1-benzothiazolopyrazoline derivatives were synthesized by the reaction of appropriately substituted chalcones and 2hydrazinobenzothiazole in ethanol. The synthesized heterocycles have been characterized on the basis of their chemical properties and spectroscopic data. These compounds were tested for biological activity against a variety of test organisms.
\end{abstract}

Keywords: Chalcones, 2-Hydrazinobenzothiazole, 3,5-Diaryl-1-benzothiazolopyrazoline, Biological activity

\section{Introduction}

Heterocycles bearing nitrogen, sulphur and thiazole moieties constitute the core structure of a number of biologically interesting compounds. Pyrazolines are an interesting group of compounds, many of which possess wide-spread pharmacological properties such as analgesic, antipyretic, antidepressant and antirheumatic activities ${ }^{1,2}$ and are also known for their pronounced anti-inflammatory activity ${ }^{3}$ and are used as potent antidiabetic agents $\mathrm{s}^{4,5}$. Recently, pyrazolines were reported as a DP-IV inhibitors and antitumor agents ${ }^{6-8}$. Some nitrogen heterocyclic compounds were reported to be antiparkinsonian ${ }^{9}$, anticancer ${ }^{10,11}$, antimicrobial ${ }^{12-15}$ and anti-inflammatory agents ${ }^{16,17}$. Due to the interesting activity of various substituted pyrazolines as biological agents considerable attention has been focused on this class. In addition, pyrazolines have played a crucial part in the development of theory in heterocyclic chemistry and also used extensively in organic synthesis ${ }^{18-22}$. Benzothiazole and its derivatives ${ }^{23-27}$ have been recognized as a class of medicinal importance. Benzothiazoles have also been screened for their antituberculostic activity ${ }^{28}$. Benzothiazole derivatives represent an extensive group of heterocyclic compounds, several of which have already 
found application in the medical sphere as medicines ${ }^{29}$ as well as in agriculture ${ }^{30,31}$. Many of 2-R substituted benzothiazoles are known as substances with antibacterial and antifungal activities ${ }^{32-34}$ and are reported also to be active as antineoplastics ${ }^{35}$ agent. Keeping in view the biological and clinical importance of benzothiazoles and pyrazolines, in this paper we report the synthesis and biological activity of new 3,5-diaryl-1-benzothiazolopyrazoline derivatives.

\section{Experimental}

\section{General procedures}

Melting points were determined by the open tube capillary method and are uncorrected. The purity of the compounds was controlled by thin layer chromatography (TLC). IR spectra were recorded as $\mathrm{KBr}$ pellets on Perkin-Elmer spectrum RX1 spectrophotometer. Carbon, hydrogen, nitrogen and sulphur were estimated by Thermo Finnigan FLASH EA 1112 elemental analyzer. ${ }^{1} \mathrm{H}-\mathrm{NMR}$ spectra were recorded on Bruker DRX-300 spectrometer at room temperature. Mass spectra were measured on JEOL SX 102/DA-6000 mass spectrometer. Substituted chalcones (1-20) were prepared according to the reported methods.

\section{Synthesis of 2-hydrazinobenzothiazole}

To a suspension of 2-aminobenzothiazole $(7.5 \mathrm{~g})$ in ethylene glycol $(40 \mathrm{~mL})$, hydrazine hydrate $(100 \%, 10 \mathrm{~mL})$ and concentrated hydrochloric acid $(10 \mathrm{~mL})$ was added at $5-6{ }^{\circ} \mathrm{C}$. The reaction mixture was refluxed for $2-3 \mathrm{~h}$ and cooled to room temperature. The reaction mixture was filtered and resulting precipitate was washed with distilled water. The resulting crude was crystallized from ethanol to obtained brownish black crystalline product.

\section{General procedure for the preparation of compounds(21-40)}

A mixture of substituted chalcone (1-20, $10.00 \mathrm{~m} \mathrm{~mol})$ and 2-hydrazinobenzothiazole (10.00 $\mathrm{m} \mathrm{mol})$ in ethanol $(50 \mathrm{~mL})$ was refluxed for $5 \mathrm{~h}$ and then cooled to room temperature. The precipitate was separated by filtration, washed with water and crystallized from a mixture of alcohol and chloroform (1:1) to obtained 3, 5-diaryl-1-benzothiazolopyrazoline derivatives (Scheme 1).

\section{3,5-Diphenyl-1-benzothiazolopyrazoline(21)}

Obtained as white crystals in $70 \%$ yield, m.p. $174-175{ }^{\circ} \mathrm{C} ;{ }^{1} \mathrm{H}$ NMR $(\delta): 3.25\left(1 \mathrm{H}\right.$,dd, $\mathrm{CH}_{2(\text { Pyraz })}$ ), $3.90\left(1 \mathrm{H}, \mathrm{dd}, \mathrm{CH}_{2 \text { (Pyraz) }}\right), 5.80\left(1 \mathrm{H}, \mathrm{dd}, \mathrm{CH}_{\text {(Pyraz) }}\right), 7.00-7.90(14 \mathrm{H}, \mathrm{m}, \mathrm{ArH})$; IR(v) max: 3400, 3030, $1600,1490,1440,1400,1320,1280,1230,1120,1020,880,810760,730700,670,540$ and 430 $\mathrm{cm}^{-1}$; MS, $m / z: 355,354,278,233,154,126,112,100,86$. Anal. Calcd. for $\mathrm{C}_{22} \mathrm{H}_{17} \mathrm{~N}_{3} \mathrm{~S}: \mathrm{C}, 74.36$; H, 4.78; N, 11.83; S, 9.02. Found: C, 74.43; H, 4.72; N, 11.90; S, 9.01\%.

\section{3(2'-Hydroxyphenyl)-5-phenyl-1-benzothiazolopyrazoline(22)}

Obtained as white crystals in $80 \%$ yield, m.p. $187-188{ }^{\circ} \mathrm{C} ;{ }^{1} \mathrm{H}$ NMR $(\delta): 3.40\left(1 \mathrm{H}\right.$,dd, $\left.\mathrm{CH}_{2 \text { (Pyraz) }}\right)$, $4.00\left(1 \mathrm{H}, \mathrm{dd}, \mathrm{CH}_{2 \text { (Pyraz) }}\right), 5.75\left(1 \mathrm{H}, \mathrm{dd}, \mathrm{CH}_{\text {(Pyraz) }}\right), 6.90-7.80(13 \mathrm{H}, \mathrm{m}, \mathrm{ArH}) ; 10.11(1 \mathrm{H}, \mathrm{s}, \mathrm{OH}) ; \mathrm{IR}$ (v) max: 3400, 3210, 3030, 3000-2500 (Stretching vibrations), 1610, 1590, 1490, 1460, 1440, 1400, 320, 1300, 1250, 1140, 1040, 1020, 880, 830, 760, 700, 660, 570, 500 and $440 \mathrm{~cm}^{-1}$; MS, $m / z: 371,370,354,294,277,170,153,128,111,102,85$. Anal. Calcd. for $\mathrm{C}_{22} \mathrm{H}_{17} \mathrm{~N}_{3} \mathrm{OS}$ : C, 71.15; H, 4.58; N, 11.32; S, 8.62. Found: C, 71.25; H, 4.45; N, 11.32; S, 8.63\%.

\section{3(4'-Chlorophenyl)-5-phenyl-1-benzothiazolopyrazoline(23)}

Obtained as white crystals in $75 \%$ yield, m.p. $165-166{ }^{\circ} \mathrm{C} ;{ }^{1} \mathrm{H}$ NMR $(\delta): 3.26\left(1 \mathrm{H}\right.$, dd, $\left.\mathrm{CH}_{2 \text { (Pyraz) }}\right)$, $3.91\left(1 \mathrm{H}, \mathrm{dd}, \mathrm{CH}_{2(\text { Pyraz })}\right), 5.81\left(1 \mathrm{H}, \mathrm{dd}, \mathrm{CH}_{(\text {(yraz) }}\right), 7.01-7.91(14 \mathrm{H}, \mathrm{m}, \mathrm{ArH})$; IR (v) max: 3400, 3030, 2900, 1600-1490, 1440, 1400, 1320, 1280, 1240, 1140, 1090, 880, 820, 750, 720, 700, 610, 530 
and $430 \mathrm{~cm}^{-1}$; MS, $\mathrm{m} / \mathrm{z}: 389,388,352,312,276,188,152,146,120,110,84$. Anal. Calcd. for $\mathrm{C}_{22} \mathrm{H}_{16} \mathrm{ClN}_{3} \mathrm{~S}$ : C, 67.86; H, 4.11; N, 10.79; S, 8.23 Found: C, 67.95; H, 4.21; N, 10.79; S, 8.22\%.

\section{3(4'-Methylphenyl)-5-phenyl-1-benzothiazolopyrazoline (24)}

Obtained as white crystals in $78 \%$ yield, m.p. $160-161{ }^{\circ} \mathrm{C} ;{ }^{1} \mathrm{H}$ NMR $(\delta): 3.24\left(1 \mathrm{H}\right.$, dd, $\left.\mathrm{CH}_{2 \text { (Pyraz) }}\right)$, $3.88\left(1 \mathrm{H}, \mathrm{dd}, \mathrm{CH}_{2(\text { Pyraz })}\right), 5.82\left(1 \mathrm{H}, \mathrm{dd}, \mathrm{CH}_{(\text {(Pyraz })}\right), 7.00-7.88(14 \mathrm{H}, \mathrm{m}, \mathrm{ArH})$; IR (v) max: 3400, 3030, 2900, 1600, 1490, 1440, 1400, 1330, 1280, 1230, 1120, 1040, 1020, 880, 810, 750, 690, 530 and $430 \mathrm{~cm}^{-1}$; MS, $\mathrm{m} / z:$ 369, 368, 354, 292, 277, 168, 153, 126, 111, 100, 85. Anal. Calcd. for $\mathrm{C}_{23} \mathrm{H}_{19} \mathrm{~N}_{3} \mathrm{~S}$ : C, 74.79; H,5.14; N, 11.38; S, 8.67. Found: C, 74.96; H, 5.08; N, 11.37; S, 8.68\%.

\section{3(4'-Methoxyphenyl)-5-phenyl-1-benzothiazolopyrazoline(25)}

Obtained as white crystals in $71 \%$ yield, m.p. $149-150{ }^{\circ} \mathrm{C} ;{ }^{1} \mathrm{H}$ NMR $(\delta): 3.30(1 \mathrm{H}, \mathrm{dd}$, $\left.\mathrm{CH}_{2 \text { (Pyraz) }}\right), 3.90\left(1 \mathrm{H}, \mathrm{dd}, \mathrm{CH}_{2 \text { (Pyraz) }}\right), 5.75\left(1 \mathrm{H}, \mathrm{dd}, \mathrm{CH}_{\text {(Pyraz) }}\right), 6.80-7.80(13 \mathrm{H}, \mathrm{m}, \mathrm{ArH}) ; 3.80$ $\left(1 \mathrm{H}, \mathrm{s},-\mathrm{OCH}_{3}\right)$; IR (v) max: 3400, 3220, 3030, 1660, 1600, 1500, 1440, 1310, 1250, 1170, 1120, 1050, 880, 830, 760, 700, 580, 530 and $430 \mathrm{~cm}^{-1}$; MS, $\mathrm{m} / z: 385,384,354,308,277$, 184, 153, 142, 126, 111, 85. Anal. Calcd. for $\mathrm{C}_{23} \mathrm{H}_{19} \mathrm{~N}_{3} \mathrm{OS}$ : C, 71.68; H, 4.93; N, 10.90; S, 8.31. Found: C, 71.73; H, 4.87; N, 10.93; S, 8.29\%.

\section{3-Phenyl-5-(2-hydroxyphenyl)-1-benzothiazolopyrazoline(26)}

Obtained as white crystals in $65 \%$ yield, m.p. $230-231{ }^{\circ} \mathrm{C} ;{ }^{1} \mathrm{H}$ NMR $(\delta): 3.60(1 \mathrm{H}, \mathrm{dd}$, $\begin{array}{lllll}\left.\mathrm{CH}_{2 \text { (Pyraz) }}\right), & 3.95\left(1 \mathrm{H}, \mathrm{dd}, \quad \mathrm{CH}_{2 \text { (Pyraz) }}\right), \quad 6.20 \quad\left(1 \mathrm{H}, \mathrm{dd}, \quad \mathrm{CH}_{\text {(Pyraz) }}\right), \quad 6.80-7.90\end{array}$ (13H,m,ArH);11.15(1H,s,-OH); IR(v)max: 3400, 3030, 3000, 2500, 1600, 1530, 1490, 1450, 1280, 1230, 1130, 1100, 1020, 890, 750, 690, 530 and $430 \mathrm{~cm}^{-1}$; MS, $\mathrm{m} / z: 371,370,354$, 294, 277, 170, 153, 128, 111, 102, 85. Anal. Calcd. for $\mathrm{C}_{22} \mathrm{H}_{17} \mathrm{~N}_{3} \mathrm{OS}: \mathrm{C}, 71.15 ; \mathrm{H}, 4.58 ; \mathrm{N}$, 11.32;S, 8.63 Found: C, 71.23; H, 4.52; N, 11.41; S, 8.65\%.

\section{3(2'-Hydroxyphenyl)-5-(2-hydroxyphenyl)-1-benzothiazolopyrazoline(27)}

Obtained as white crystals in $72 \%$ yield, m.p. $242-243{ }^{\circ} \mathrm{C} ;{ }^{1} \mathrm{H}$ NMR $(\delta): 3.62\left(1 \mathrm{H}, \mathrm{dd}, \mathrm{CH}_{2 \text { (Pyraz) }}\right)$, $3.92\left(1 \mathrm{H}, \mathrm{dd}, \mathrm{CH}_{2 \text { (Pyraz) }}\right), 6.21\left(1 \mathrm{H}, \mathrm{dd}, \mathrm{CH}_{\text {(Pyraz) }}\right), 6.81-7.92(13 \mathrm{H}, \mathrm{m}, \mathrm{ArH}) ; 11.16(1 \mathrm{H}, \mathrm{s},-\mathrm{OH})$; IR(v)max: 3100, 3030, 3000-2500, 1600, 1540, 1450, 1280, 1120, 1100, 1020, 890, 870, 750, 730, 690, 680, 540 and $430 \mathrm{~cm}^{-1}$; MS, $\mathrm{m} / z: 387,386,370,293,169,127,101$. Anal. Calcd. for $\mathrm{C}_{22} \mathrm{H}_{17} \mathrm{~N}_{3} \mathrm{O}_{2} \mathrm{~S}: \mathrm{C}, 68.21 ; \mathrm{H}, 4.39 ; \mathrm{N}, 10.8 ; \mathrm{S}, 8.27$ Found: C, 68.28; H, 4.39; N, 10.85; S, 8.28\%.

\section{3(4'-Chlorophenyl)-5-(2-hydroxyphenyl)-1-benzothiazolopyrazoline(28)}

Obtained as white crystals in $76 \%$ yield, m.p. $250-251{ }^{\circ} \mathrm{C} ;{ }^{1} \mathrm{H}$ NMR $(\delta): 3.60(1 \mathrm{H}, \mathrm{dd}$, $\left.\mathrm{CH}_{2 \text { (Pyraz) }}\right), \quad 3.95\left(1 \mathrm{H}, \mathrm{dd}, \mathrm{CH}_{2 \text { (Pyraz) }}\right), 6.20\left(1 \mathrm{H}, \mathrm{dd}, \mathrm{CH}_{\text {(Pyraz) }}\right), 6.80-7.90(13 \mathrm{H}, \mathrm{m}, \mathrm{ArH}) ; 11.14$ (1H,s,-OH); IR(v)max: 3400, 3030, 3000-2500, 1600, 1540, 1490, 1450, 1400, 1320, 1280 , 1240, 1130, 1100, 1010, 890, 830, 750, 530 and $430 \mathrm{~cm}^{-1}$; MS, $m / z: 405,404,369,334,392$, 257, 168, 133, 126, 100, 91, 65. Anal. Calcd. for $\mathrm{C}_{22} \mathrm{H}_{16} \mathrm{ClN}_{3} \mathrm{OS}: \mathrm{C}, 65.18 ; \mathrm{H}, 3.95 ; \mathrm{N}$, 10.37; S, 7.90. Found: C, 65.23; H, 3.93; N, 10.42; S, 7.92\%.

\section{3(4'-Methylphenyl)-5-(2-hydroxyphenyl)-1-benzothiazolopyrazoline(29)}

Obtained as white crystals in $65 \%$ yield, m.p. $229-230{ }^{\circ} \mathrm{C} ;{ }^{1} \mathrm{H}$ NMR $(\delta): 3.59(1 \mathrm{H}, \mathrm{dd}$, $\left.\mathrm{CH}_{2 \text { (Pyraz) }}\right), 3.94\left(1 \mathrm{H}, \mathrm{dd}, \mathrm{CH}_{2 \text { (Pyraz) }}\right), 6.22\left(1 \mathrm{H}, \mathrm{dd} \mathrm{CH}_{(\text {Pyraz })}\right), 6.80-7.89(13 \mathrm{H}, \mathrm{m}, \mathrm{ArH}) ; 11.15(1 \mathrm{H}, \mathrm{s},-$ OH); IR(v)max: 3400 3030, 3000-2500, 600, 1550, 1490, 1450, 1380,1 320, 1280, 1230, 1180 , 1100, 1050, 1030, 890, 810, 750, 720, 690, 530 and $430 \mathrm{~cm}^{-1}$; MS, $\mathrm{m} / z: 385,384,370,368$, 293, 291, 169, 167, 127, 125, 101, 99. Anal. Calcd. For $\mathrm{C}_{23} \mathrm{H}_{19} \mathrm{~N}_{3} \mathrm{OS}: \mathrm{C}, 71.68 ; \mathrm{H}, 4.93$; N, 10.90; S, 8.31. Found: C, 71.78; H, 4.89; N, 10.92; S, 8.35\%. 
3(4'-Methoxyphenyl)-5-(2-hydroxyphenyl)-1-benzothiazolopyrazoline(30)

Obtained as white crystals in $65 \%$ yield, m.p. $247-248{ }^{\circ} \mathrm{C} ;{ }^{1} \mathrm{H}$ NMR $(\delta): 3.64(1 \mathrm{H}, \mathrm{dd}$, $\left.\mathrm{CH}_{2 \text { (Pyraz) }}\right), 3.96\left(1 \mathrm{H}, \mathrm{dd}, \mathrm{CH}_{2 \text { (Pyraz) }}\right), 6.21\left(1 \mathrm{H}, \mathrm{dd}, \mathrm{CH}_{\text {(Pyraz) }}\right), 6.82-7.90(13 \mathrm{H}, \mathrm{m}, \mathrm{ArH}) ; 11.16$ (1H,s,-OH); IR(v)max: 3400, 3030, 3000-2500, 1600, 1540, 1450, 1320, 1250, 1170, 1120, 1030, 880, 830, 750, 630, 530 and $430 \mathrm{~cm}^{-1}$; MS, $\mathrm{m} / \mathrm{z}: 401,400,384,370,307,293$, 183, 169, 141, 127, 115, 101. Anal. Calcd. for $\mathrm{C}_{23} \mathrm{H}_{19} \mathrm{~N}_{3} \mathrm{O}_{2} \mathrm{~S}: \mathrm{C}, 68.93 ; \mathrm{H}, 4.73 ; \mathrm{N}, 10.47 ; \mathrm{S}$, 7.98. Found: C, 68.93; H, 4.71; N, 10.43; S, 7.99\%.

\section{3(2'-Hydroxyphenyl)-5-(4-methoxyphenyl)-1-benzothiazolopyrazoline(31)}

Obtained as white crystals in $78 \%$ yield, m.p. $185-186{ }^{\circ} \mathrm{C} ;{ }^{1} \mathrm{H}$ NMR $(\delta): 3.35(1 \mathrm{H}, \mathrm{dd}$, $\left.\mathrm{CH}_{2 \text { (Pyraz) }}\right), 3.75\left(3 \mathrm{H}, \mathrm{s},-\mathrm{OCH}_{3}\right) ; 3.95\left(1 \mathrm{H}, \mathrm{dd}, \mathrm{CH}_{2 \text { (Pyraz) }}\right), 5.70\left(1 \mathrm{H}, \mathrm{dd}, \mathrm{CH}_{(\text {Pyraz })}\right), 6.80-7.80$ (12H,m,ArH); 10.10(1H,s,-OH); IR(v)max: 3200, 3030,3000-2500, 1610, 1590, 1490, 1450, 1400, 1320, 1290, 1240, 1170, 1140, 1100, 1030, 880, 830, 820, 760, 730, 660, 640, 570, 550, 500 and $430 \mathrm{~cm}^{-1}$; MS, $\mathrm{m} / \mathrm{z}: 401,400,384,370,307,293,183,169,141,127,115$, 101. Anal. Calcd. for $\mathrm{C}_{23} \mathrm{H}_{19} \mathrm{~N}_{3} \mathrm{O}_{2} \mathrm{~S}: \mathrm{C}, 68.82 ; \mathrm{H}, 4.73 ; \mathrm{N}, 10.47 ; \mathrm{S}, 7.98$. Found: $\mathrm{C}, 68.93$; $\mathrm{H}, 4.71 ; \mathrm{N}, 10.43 ; \mathrm{S}, 7.95 \%$.

\section{3(4'-Chlorophenyl)-5-(4-methoxyphenyl)-1-benzothiazolopyrazoline(32)}

Obtained as white crystals in $65 \%$ yield, m.p. $158-159{ }^{\circ} \mathrm{C} ;{ }^{1} \mathrm{H}$ NMR $(\delta): 3.35(1 \mathrm{H}, \mathrm{dd}$, $\left.\mathrm{CH}_{2 \text { (Pyraz) })}\right), 3.75\left(3 \mathrm{H}, \mathrm{s},-\mathrm{OCH}_{3}\right) ; 3.94\left(1 \mathrm{H}, \mathrm{dd}, \mathrm{CH}_{2 \text { (Pyraz) })}\right), 5.71\left(1 \mathrm{H}, \mathrm{dd} \mathrm{CH}_{(\text {Pyraz })}\right), 6.80-7.82$ (12H,m,ArH);10.12(1H,s,-OH); IR(v)max: 3400, 3040, 2900, 1610, 1510, 1430, 1250, 1180 , 1090, 1030, 880, 820, 75, 630, 540 and $430 \mathrm{~cm}^{-1}$; MS, $\mathrm{m} / \mathrm{z}: 419,418,388,383,306,264$, 187, 182, 147, 140, 119, 114. Anal. Calcd. for $\mathrm{C}_{23} \mathrm{H}_{18} \mathrm{ClN}_{3} \mathrm{OS}: \mathrm{C}, 65.87 ; \mathrm{H}, 4.53 ; \mathrm{N}, 10.02$; S, 7.64. Found: C, 65.90; H, 4.51; N, 10.10; S, 7.62\%.

\section{3(4'-Methylphenyl)-5-(4-methoxyphenyl)-1-benzothiazolopyrazoline(33)}

Obtained as white crystals in $68 \%$ yield, m.p. $175-176{ }^{\circ} \mathrm{C} ;{ }^{1} \mathrm{H}$ NMR $(\delta): 3.36(1 \mathrm{H}, \mathrm{dd}$, $\left.\mathrm{CH}_{2 \text { (Pyraz) }}\right)$, $3.76\left(3 \mathrm{H}\right.$, s, $\left.-\mathrm{OCH}_{3}\right) ; 3.96\left(1 \mathrm{H}, \mathrm{dd} \mathrm{CH}_{2 \text { (Pyraz) }}\right), 5.73\left(1 \mathrm{H}, \mathrm{dd}, \mathrm{CH}_{\text {(Pyraz) }}\right), 6.82-$ 7.80(12H,m,ArH); 10.14(1H,s,-OH); IR(v)max: 3400, 3030, 2940, 2820, 1600, 1530, 1490, $1440,1320,1250,1180,1120,1030,880,830,750,720,690,670,530$ and $430 \mathrm{~cm}^{-1} ; \mathrm{MS}, \mathrm{m} / z$ : $399,398,384,383,368,306,219,182,167,140,125,114,99$. Anal. Calcd. for $\mathrm{C}_{24} \mathrm{H}_{21} \mathrm{~N}_{3} \mathrm{OS}$ : C, 72.18; H, 5.26; N, 10.52 S, 8.02. Found: C, 72.20; H, 5.23; N, 10.52 S, 8.04\%.

\section{3-Phenyl-5-(4-chlorophenyl)-1-benzothiazolopyrazoline(34)}

Obtained as white crystals in $74 \%$ yield, m.p. $145-146{ }^{\circ} \mathrm{C} ;{ }^{1} \mathrm{H}$ NMR $(\delta): 3.25(1 \mathrm{H}, \mathrm{dd}$, $\left.\mathrm{CH}_{2 \text { (Pyraz) }}\right), 3.90\left(1 \mathrm{H}, \mathrm{dd}, \mathrm{CH}_{2 \text { (Pyraz) }}\right), 5.75\left(1 \mathrm{H}, \mathrm{dd}, \mathrm{CH}_{(\text {(Pyraz) }}\right), 7.00-7.80(13 \mathrm{H}, \mathrm{m}, \mathrm{ArH})$; IR(v)max: $3400,3030,2900,1650,1600,1530,1490,1440,1400,1320,1280,1230,1130,1100$, 1080, 1030, 1000, 880, 820, 780, 750, 730, 690, 670, 560 and $430 \mathrm{~cm}^{-1} ; \mathrm{MS}, \mathrm{m} / z: 389,388$, $353,312,311,276,190,154,148,122,112,86$. Anal. Calcd. for $\mathrm{C}_{22} \mathrm{H}_{16} \mathrm{ClN}_{3} \mathrm{~S}: \mathrm{C}, 67.86 ; \mathrm{H}$, $4.11 ; \mathrm{N}, 10.79 ; \mathrm{S}, 8.23$. Found: C, 68.02; H, 4.09; N, 10.76; S, 8.20\%.

\section{3(2'-Hydroxyphenyl)-5-(4-chlorophenyl)-1-benzothiazolopyrazoline(35)}

Obtained as white crystals in $71 \%$ yield, m.p. $\left.149-150{ }^{\circ} \mathrm{C} ;{ }^{1} \mathrm{H} \mathrm{NMR}(\delta): 3.26\left(1 \mathrm{H}, \mathrm{dd}, \mathrm{CH}_{2(\mathrm{Pyraz}}\right)\right)$, 3.91(1H,dd, $\left.\left.\left.\quad \mathrm{CH}_{2(\text { (Praz })}\right)\right), \quad 5.76\left(1 \mathrm{H}, \mathrm{dd}, \quad \mathrm{CH}_{(\text {(Praz })}\right)\right), 7.02-7.84 \quad(13 \mathrm{H}, \mathrm{m}, \mathrm{ArH}) ; 11.15(1 \mathrm{H}, \mathrm{s},-\mathrm{OH})$; IR(v)max: 3400, 3030, 3000-2500, 1600, 1530, 1490, 1440, 1400, 1320, 1280, 1230, 1130, 1100, 1080, 1000, 880, 830, 780, 750, 720, 690, 670, 550 and $430 \mathrm{~cm}^{-1}$; MS, $\mathrm{m} / \mathrm{z}: 405,404$, 388, 387, 293, 265, 223, 171, 197, 129, 103. Anal. Calcd. for $\mathrm{C}_{22} \mathrm{H}_{16} \mathrm{ClN}_{3} \mathrm{OS}: \mathrm{C}, 65.18 ; \mathrm{H}, 3.95$; N, 10.37; S, 7.90. Found: C, 68.20; H, 3.98; N, 10.42; S, 7.88\%. 
3(4'-Chlorophenyl)-5-(4-chlorophenyl)-1-benzothiazolopyrazoline(36)

Obtained as white crystals in $72 \%$ yield, m.p. 155-156 ${ }^{\circ} \mathrm{C} ;{ }^{1} \mathrm{H}$ NMR $(\delta): 3.24(1 \mathrm{H}, \mathrm{dd}$, $\left.\left.\mathrm{CH}_{2 \text { (Pyraz) }}\right)\right), \quad 3.95\left(1 \mathrm{H}, \mathrm{dd}, \quad \mathrm{CH}_{2 \text { (Pyraz) }}\right), \quad 5.77\left(1 \mathrm{H}, \mathrm{dd}, \quad \mathrm{CH}_{(\text {(Pyraz })}\right), \quad 7.04-7.85 \quad(13 \mathrm{H}, \mathrm{m}, \mathrm{ArH})$; IR(v)max: 3400, 3030, 2900, 1880, 1660, 1240, 1210, 1150, 1020, 880, 840, 780, 750, 730, 700, 630, 600, 530, 520, 480 and $430 \mathrm{~cm}^{-1}$; MS, $\mathrm{m} / \mathrm{z}: 423,422,387,310,186,144$, 118. Anal. Calcd. for $\mathrm{C}_{22} \mathrm{H}_{15} \mathrm{Cl}_{2} \mathrm{~N}_{3} \mathrm{~S}: \mathrm{C}, 62.41 ; \mathrm{H}, 3.54 ; \mathrm{N}, 9.92 ; \mathrm{S}, 7.57$. Found: C, 62.49; H, $3.58 ; \mathrm{N}, 9.92 ; \mathrm{S}, 7.55 \%$.

\section{3(4'-Methylphenyl)-5-(4-chlorophenyl)-1-benzothiazolopyrazoline(37)}

Obtained as white crystals in 78\% yield, m.p. 195-196 ${ }^{\circ} \mathrm{C} ;{ }^{1} \mathrm{H}$ NMR $(\delta): 3.23$ (1H,dd, $\left.\mathrm{CH}_{2 \text { (Pyraz) }}\right), 3.89\left(1 \mathrm{H}, \mathrm{dd}, \mathrm{CH}_{2 \text { (Pyraz) }}\right), 5.75\left(1 \mathrm{H}, \mathrm{dd}, \mathrm{CH}_{(\text {(Pyraz) }}\right), 7.00-7.85$ (13H,m,ArH); IR(v)max: $3400,3030,2900,1600,1530,1490,1440,1400,1330,1280,1230,1130,1100,1080$, $1040,1010,880,820,780,750,720,690,670,600,560$ and $430 \mathrm{~cm}^{-1} ; \mathrm{MS}, \mathrm{m} / z: 403,402$, $388,368,311,310,291,187,169,145,127,119,101$. Anal. Calcd. For $\mathrm{C}_{23} \mathrm{H}_{18} \mathrm{ClN}_{3} \mathrm{~S}: \mathrm{C}$, 68.48; H, 4.46; N, 10.42;S, 7.94. Found: C, 68.52; H, 4.49; N, 10.49; S, 7.93\%.

\section{3(4'-Methoxyphenyl)-5-(4-chlorophenyl)-1-benzothiazolopyrazoline(38)}

Obtained as white crystals in $68 \%$ yield, m.p. $128-129{ }^{\circ} \mathrm{C} ;{ }^{1} \mathrm{H}$ NMR $(\delta): 3.24(1 \mathrm{H}, \mathrm{dd}$, $\left.\mathrm{CH}_{2 \text { (Pyraz) }}\right), 3.93\left(1 \mathrm{H}, \mathrm{dd}, \mathrm{CH}_{2(\text { (Pyraz) }}\right), 5.76\left(1 \mathrm{H}, \mathrm{dd}, \mathrm{CH}_{(\text {Pyraz })}\right), 7.05-7.86(13 \mathrm{H}, \mathrm{m}, \mathrm{ArH}) ; 3.76(3 \mathrm{H}$, s, $-\mathrm{OCH}_{3}$ ); IR(v)max: 3400, 3030, 1650, 1600, 1510, 1440, 1400, 1250, 1180, 1090, 1040, 880, 750, 690, 670, 550 and $430 \mathrm{~cm}^{-1}$; MS, $\mathrm{m} / z: 419,418,388,383,306,264,222,196$, 182, 140, 114. Anal. Calcd. For $\mathrm{C}_{23} \mathrm{H}_{18} \mathrm{ClN}_{3} \mathrm{OS}$ : C, 65.87; H, 4.29; N, 10.11; S, 7.64. Found: C, 65.91; H, 4.32; N, 10.13; S, 7.65\%.

\section{3-(4'-Chlorophenyl-5-(3,4-dimethoxyphenyl)-1-benzothiazolopyrazoline(39)}

Obtained as white crystals in $78 \%$ yield, m.p. $144-145{ }^{\circ} \mathrm{C} ;{ }^{1} \mathrm{H}$ NMR $(\delta): 3.28(1 \mathrm{H}, \mathrm{dd}$, $\left.\mathrm{CH}_{2 \text { (Pyraz) }}\right), 3.96\left(1 \mathrm{H}, \mathrm{dd}, \mathrm{CH}_{2 \text { (Pyraz) }}\right), 5.27\left(1 \mathrm{H}, \mathrm{dd}, \mathrm{CH}_{\text {(Pyraz) }}\right), 6.85-7.76(11 \mathrm{H}, \mathrm{m}, \mathrm{ArH}), 3.85(6 \mathrm{H}$, 2-s, - $\mathrm{OCH}_{3}$ ); IR(v)max: 3400, 3030,2920, 2820, 1600, 1530, 1490, 1440,1260, 1230, 1140, 1090, 1030, 900, 830, 750, 730, 700, 640, 590 and $430 \mathrm{~cm}^{-1}$; MS, $\mathrm{m} / z: 449,448,417,414$, $386,337,309,213,185,171,145,143,117,114$. Anal. Calcd. For $\mathrm{C}_{24} \mathrm{H}_{20} \mathrm{ClN}_{3} \mathrm{O}_{2} \mathrm{~S}: \mathrm{C}$, 64.14; H, 4.45; N, 9.35; S, 7.13. Found: C, 64.18; H, 4.44; N, 9.42; S, 7.15\%.

\section{3-Phenyl-5-(3,4-dimethoxyphenyl)-1-benzothiazolopyrazoline(40)}

Obtained as white crystals in $80 \%$ yield, m.p. $162-163{ }^{\circ} \mathrm{C} ;{ }^{1} \mathrm{H}$ NMR $(\delta): 3.30(1 \mathrm{H}, \mathrm{dd}$, $\left.\mathrm{CH}_{2 \text { (Pyraz) }}\right), 3.90\left(1 \mathrm{H}, \mathrm{dd}, \mathrm{CH}_{2(\text { (Pyraz) }}\right), 5.25\left(1 \mathrm{H}, \mathrm{dd}, \mathrm{CH}_{\text {(Pyraz) }}\right), 6.70-7.80(11 \mathrm{H}, \mathrm{m}, \mathrm{ArH}), 3.80(6 \mathrm{H}$, 2-s, - $\mathrm{OCH}_{3}$ ); IR(v)max: 3400, 3030,2920, 2820, 1600, 1530, 1490, 1440, 1320, 1260, 1230 , 1140, 1030, 900, 820, 760, 690, 670, 640, 540 and $430 \mathrm{~cm}^{-1}$; MS, $\mathrm{m} / z: 415,414,399,384$, $338,307,214,183,172,146,141,115$. Anal. Calcd. For $\mathrm{C}_{24} \mathrm{H}_{21} \mathrm{~N}_{3} \mathrm{O}_{2} \mathrm{~S}: \mathrm{C}, 69.42 ; \mathrm{H}, 5.05 ; \mathrm{N}$, 10.18; S, 7.71. Found: C, 69.39; H, 5.06; N, 10.12; S, 7.70\%.

\section{Results and Discussion}

Formation of 3,5-diarylpyrazoline derivatives by the reaction of $\alpha, \beta$-unsaturated ketones and hydrazines may take place under various reaction conditions using ethanol ${ }^{36}$ as solvent. In our present case to obtained 3,5-diaryl-1-benzothiazolopyrazoline derivatives (21-40) a mixture of substituted chalcone (1-20, $10.00 \mathrm{~m} \mathrm{~mol})$ and 2-hydrazinobenzothiazole $(10.00 \mathrm{~m}$ $\mathrm{mol})$ in ethanol $(50 \mathrm{~mL})$ was refluxed for $5 \mathrm{~h}$ and then cooled to room temperature. The precipitate was separated by filtration, washed with water and crystallized from a mixture of alcohol and chloroform (1:1) (Scheme 1). Substituted chalcones (1-20) were prepared 
according to the reported methods. Structures of all new compounds (21-40) have been elucidated by elemental analyses, ${ }^{1} \mathrm{H}$ NMR, Mass spectral and IR measurements. In the IR spectra of 3,5-diaryl-1-benzothiazolopyrazoline derivatives (21-40) there is no carbonyl absorption but absorption bands in the region $1600-1450 \mathrm{~cm}^{-1}$ due to the presence of $\mathrm{C}=\mathrm{C}$ and $\mathrm{C}=\mathrm{N}$ stretching vibrations. Stretching vibrations due to the intramolecular hydrogen bonding of $-\mathrm{OH}$ group gave absorption in the region $3000-2500 \mathrm{~cm}^{-1}$.

In addition to aromatic protons, the ${ }^{1} \mathrm{H}$ NMR spectra of these compounds exhibit double doublets for each $-\mathrm{CH}_{2}$ proton between $\delta 3.23$ to 3.64 and $\delta 3.88$ to 4.00 and double doublet between $\delta 5.25$ to 6.22 for $-\mathrm{CH}$ proton of the pyrazoline nucleus (ABX) system.<smiles>NNc1nc2ccccc2s1</smiles>

Substituded Chalcones (1-20) 2-Hydrazinobenzothiazole<smiles>Brc1ccc(C2=NN(c3nc4ccccc4s3)C(c3ccc(Br)c(Br)c3)C2)cc1</smiles>

3,5-Diaryl-1-benzothiazolopyrazoline (21-40)

21: $R_{1}=R_{2}=R^{3}=R^{4}=R^{5}=H$

22: $\mathrm{R}_{2}=\mathrm{OH}, \mathrm{R}_{1}=\mathrm{R}_{3}=\mathrm{R}_{4}=\mathrm{R}_{5}=\mathrm{H}$

23: $\mathrm{R}_{1}=\mathrm{Cl}, \mathrm{R}_{2}=\mathrm{R}_{3}=\mathrm{R}_{4}=\mathrm{R}_{5}=\mathrm{H}$

24: $\mathrm{R}_{1}=\mathrm{CH}_{3}, \mathrm{R}_{2}=\mathrm{R}_{3}=\mathrm{R}_{4}=\mathrm{R}_{5}=\mathrm{H}$

25: $\mathrm{R}_{1}=\mathrm{OCH}_{3}, \mathrm{R}_{2}=\mathrm{R}_{3}=\mathrm{R}_{4}=\mathrm{R}_{5}=\mathrm{H}$

26: $\mathrm{R}_{3}=\mathrm{OH}, \mathrm{R}_{2}=\mathrm{R}_{3}=\mathrm{R}_{4}=\mathrm{R}_{5}=\mathrm{H}$

27: $\mathrm{R}_{1}=\mathrm{R}_{4}=\mathrm{R}_{5}=\mathrm{H}, \mathrm{R}_{2}=\mathrm{R}_{3}=\mathrm{OH}$

28: $\mathrm{R}_{1}=\mathrm{Cl}, \mathrm{R}_{2}=\mathrm{R}_{4}=\mathrm{R}_{5}=\mathrm{H},=\mathrm{R}_{3}=\mathrm{OH}$

29: $\mathrm{R}_{1}=\mathrm{CH}_{3}, \mathrm{R}_{2}=\mathrm{R}_{4}=\mathrm{R}_{5}=\mathrm{H}, \mathrm{R}_{3}=\mathrm{OH}$

30: $\mathrm{R}_{1}=\mathrm{OCH}_{3}, \mathrm{R}_{2}=\mathrm{R}_{4}=\mathrm{R}_{5}=\mathrm{H}, \mathrm{R}_{3}=\mathrm{OH}$
31: $\mathrm{R}_{1}=\mathrm{R}_{3}=\mathrm{R}_{4}=\mathrm{H}, \mathrm{R}_{2}=\mathrm{OH}, \mathrm{R}_{5}=\mathrm{OCH}_{3}$

32: $\mathrm{R}_{1}=\mathrm{Cl}, \mathrm{R}_{2}=\mathrm{R}_{3}=\mathrm{R}_{4}=\mathrm{H}, \mathrm{R}_{5}=\mathrm{OCH}_{3}$

33: $\mathrm{R}_{1}=\mathrm{CH}_{3}, \mathrm{R}_{2}=\mathrm{R}_{3}=\mathrm{R}_{4}=\mathrm{H}, \mathrm{R}_{5}=\mathrm{OCH}_{3}$

34: $\mathrm{R}_{1}=\mathrm{R}_{2}=\mathrm{R}_{3}=\mathrm{R}_{4}=\mathrm{H}, \mathrm{R}_{5}=\mathrm{Cl}$

35: $\mathrm{R}_{1}=\mathrm{R}_{3}=\mathrm{R}_{4}=\mathrm{H}, \mathrm{R}_{2}=\mathrm{OH}, \mathrm{R}_{5}=\mathrm{Cl}$

36: $\mathrm{R}_{1}=\mathrm{R}_{5}=\mathrm{Cl}, \mathrm{R}_{2}=\mathrm{R}_{3}=\mathrm{R}_{4}=\mathrm{H}$

37: $\mathrm{R}_{1}=\mathrm{CH}_{3}, \mathrm{R}_{2}=\mathrm{R}_{3}=\mathrm{R}_{4}=\mathrm{H}, \mathrm{R}_{5}=\mathrm{Cl}$

38: $\mathrm{R}_{1}=\mathrm{OCH}_{3}, \mathrm{R}_{2}=\mathrm{R}_{3}=\mathrm{R}_{4}=\mathrm{H}, \mathrm{R}_{5}=\mathrm{Cl}$

39: $\mathrm{R}_{1}=\mathrm{Cl}, \mathrm{R}_{2}=\mathrm{R}_{3}=\mathrm{H}, \mathrm{R}_{4}=\mathrm{R}_{5}=\mathrm{OCH}_{3}$

40: $\mathrm{R}_{1}=\mathrm{R}_{2}=\mathrm{R}_{3}=\mathrm{H}, \mathrm{R}_{4}=\mathrm{R}_{5}=\mathrm{OCH}_{3}$

Scheme 1. Synthesis of 3,5-diaryl-1-benzothiazolopyrazoline derivatives.

\section{Biological activity}

The synthesized 3,5-diaryl-1-benzothiazolopyrazoline derivatives (21-40) have been subjected to in vitro antimicrobial activity against various plant and human pathogenic bacteria and fungi.

Antimicrobial activity was carried out against Gram negative rod $P$. fluorescence, E.coli, $P$. vulgariaus and A. faecalis, gram positive rod B. subtilis and gram positive coccus S. epidermidis, C. albicans, C. cladosporoides, A. niger and C. lunata all are soil fungi obtained from standard cultures. The results are summarized in the Table 1. 
Table 1. Antimicrobial activity of 3,5-diaryl-1-benzothiazolopyrazoline derivatives.

\begin{tabular}{lllllllllll}
\hline & \multicolumn{1}{c}{} & \multicolumn{7}{c}{ Culture } \\
\hline Comp No A & B & C & D & E & F & G & H & I & J \\
\hline $\mathbf{2 1}$ & - & - & - & - & - & - & - & - & - & - \\
$\mathbf{2 2}$ & - & - & - & - & - & - & 6 & - & - & - \\
$\mathbf{2 3}$ & 22 & 15 & 10 & 14 & 14 & 8 & 9 & 9 & 7 & 8 \\
$\mathbf{2 4}$ & - & - & - & - & - & - & - & 20 & - & 21 \\
$\mathbf{2 5}$ & - & - & - & - & - & - & - & - & - & - \\
$\mathbf{2 6}$ & - & - & - & - & - & - & - & - & - & - \\
$\mathbf{2 7}$ & - & - & - & - & - & - & 8 & - & - & - \\
$\mathbf{2 8}$ & 20 & 13 & 10 & 13 & 12 & 6 & 8 & 8 & 10 & 10 \\
$\mathbf{2 9}$ & - & - & - & - & - & - & 9 & 21 & - & 20 \\
$\mathbf{3 0}$ & - & - & - & - & - & - & 8 & 20 & - & 20 \\
$\mathbf{3 1}$ & - & - & - & - & - & - & 7 & 13 & - & - \\
$\mathbf{3 2}$ & 21 & 14 & 11 & 12 & 13 & 7 & 10 & 14 & 8 & 7 \\
$\mathbf{3 3}$ & - & - & - & - & - & - & - & 20 & - & 20 \\
$\mathbf{3 4}$ & - & - & - & - & - & - & - & - & - & - \\
$\mathbf{3 5}$ & - & - & - & - & - & - & 9 & 8 & - & 8 \\
$\mathbf{3 6}$ & 20 & 13 & 10 & 13 & 12 & 6 & 8 & 7 & 8 & 7 \\
$\mathbf{3 7}$ & 21 & 14 & 11 & 12 & 13 & 7 & 9 & 8 & 7 & 9 \\
$\mathbf{3 8}$ & 22 & 15 & 10 & 14 & 14 & 6 & 9 & 8 & 8 & 7 \\
$\mathbf{3 9}$ & 20 & 13 & 11 & 13 & 13 & 8 & 8 & 8 & 8 & 8 \\
$\mathbf{4 0}$ & - & - & - & - & - & - & - & - & - & - \\
\hline
\end{tabular}

(Diameter of inhibition zone measured in $\mathrm{mm}$, paper disc $5 \mathrm{~mm}$, inhibition zone measured excluding paper disc diameter)

$A=P$. fluorescence $; B=$ E.Coli $; \quad C=P$. vulgariaus; $\quad D=$ A. faecalis; $E=B$. subtilis;

$F=S$. epidermidis; $G=C$. albicans; $H=C$. cladosporoides; $I=A$. niger; $\quad J=C$. lunata

The compounds $\mathbf{2 3}, \mathbf{2 8}, \mathbf{3 2}, \mathbf{3 6}, \mathbf{3 7}, \mathbf{3 8}, \mathbf{3 9}$ showed broad range of activity as inhibition zones were found against all plant and human bacteria and fungi. Compound 22, 26, 29, 30 , 31 and 35 possess moderate activity against $C$. albicans but compound 24, 29, 30 and 33 shows higher activity against $C$. cladosporoides and $C$. lunata.

It can be concluded from the observation that these 3,5-diaryl-1benzothiazolopyrazoline derivatives (21-40) have antimicrobial activity. Out of twenty compounds 23, 28, 32, 36, 37, 38 and 39 possessed wide range of activity. Compounds 22, 24, 27, 29, 30, 31, and 33 showed only antifungal activity. In general these 3,5-diaryl-1benzothiazolopyrazoline derivatives showed sufficient antimicrobial activity.

In conclusion, we have synthesized a systematically substituted series of new 3,5-diaryl1-benzothiazolopyrazoline derivatives for structure-activity relationship studies. These substituted benzothiazolopyrazolines are very stable compounds, which renders them beneficial substances for biological or pharmacological trials.

\section{Acknowledgements}

Authors are thankful to School of Studies in Chemistry, Vikram University, Ujjain (India), RSIC, CDRI, Lucknow (India), SAIF, Indian Institute of Technology, Bombay, Mumbai (India), Mahakal Institute of Pharmaceutical Studies, Ujjain (India) for providing spectral and analytical data. 


\section{References}

1. Jung J C, Watkins E B and Avery M A, Heterocycles, 2005, 65, 77.

2. Palaska E, Aytemir M, Uzabay T and Erol D, Eur J Med Chem., 2001, 36, 539.

3. Bansal E, Shrivastava V K and Kumar A, Eur J Med Chem., 2001, 36, 81.

4. Ahn J H, Kim H M, Jung S H, Kang S K, Kim K R, Rhee S D, Yong S D, Cheon H G and Kim S S, Bioorg Med Chem Lett., 2004, 14, 4461.

5. Villhauer E B, Brinkman J A, Naderi C B, Dunning B E, Mangold B L, Mone M D, Russell M E, Weldon S C and Hughes T E, J Med Chem., 2002, 45, 2362.

6. Amr A E, Indian J Heterocycl Chem., 2000, 10, 49.

7. Hamman A G, Fahmy A F M, Amr A E and Mohamad A M Indian J Chem., 2003, 42(B), 1985.

8. Hamman A G, Abdel Hafez N A, Midura W H. and Mikolajczyk M, Z Naturforsch., 2000, 55(B), 417.

9. Amr A E, Hegab M I, Ibrahim A A and Abdalah M M, Monatsch Chem., 2003, 134, 1395.

10. Amr A E and Abou-Ghalia M H, Amino Acids, 2004, 26, 283.

11. Brana M F, Castellano J M, Mpran M, Perez de Vega M J, Gian X D, Romerdahl C A and Keihauer G, Eur Med Chem., 1995, 30, 235.

12. Hammam A G, Sharaf M A. and Abdel-Hafez N A, Indian J Chem., 2001, 40B, 213.

13. Amr A E, Mohamed A M and Ibrahim A A, Z Naturforsch, 2003, 58b, 861.

14. Amr A E, Abdel-Salam O I, Attia A and Stibor I, Collect Czech Chem Commun., 1999, 64, 288.

15. Attia A, Abdel-Salam O I, Amr A E, Stibor I and Budesinsky M, Egypt J Chem., 2000, 43, 187.

16. Fahmy H H and Soliman G A, Arch Pharm Res., 2001, 24,180.

17. Abou-Ghalia M H, Amr A. E and Abdalah M M, Z Naturforsch, 2003, 58b, 903.

18. Tomilovi Yu V, Okonnishnikova G P, Shulishov E V and Nefedov O M, Russ Chem Bt., 1995, 44, 2114.

19. Klimova E I, Marcos M, Klimova T B, Cecilio A T, Ruben A T and Lena R R, Organometallic Chem., 1999, 585, 106.

20. Bhaskarreddy D, Padmaja A, Ramanareddy P V and Seenaiah B, Sulfur Lett., 1993, 16, 227.

21. Padmavathi V, Sumathi R P, Chandrasekhar B N and Bhaskarreddy D, J Chem Research, 1999, 610.

22. Bhaskarreddy D, Chandrasekhar B N, Padmavathi V and Sumathi R P, Synthesis, 1998, 491

23. Mahran M A, Abdel-Hamid H and El-Ashry E.-S H, Alexandria J Pharma Sci., 2007, 2(1), 7.

24. Russo F, Romeo G, Santagati N A, Caruso A, Cutuli V and Amore D, Eur J Med Chem., 1994, 29, 569.

25. Baltork I M, Khosropour A R and Hojati S F, Monatshefte fur Chemie - Chemical Monthly, 2007, 138, 663.

26. Katsura Y, Inoue Y, Nishino S, Tomoi M and Takasugi H, Chem Pharm Bull (Tokyo), 1992, 40, 1818.

27. Kuhler T C, Swanson M, Shcherbuchin V, Larsson H, Mellgard B and Sjostrom J E, J Med Chem., 1998, 41, 1777.

28. Krasovskii A N, Grin A N, Soroka I. I., Kochergin P M and Bogamyreva E I, Pharma Chem J., 1977, 11(7), 900. 
29. Kashiyama E, Hutchinson I and Chua M S, J Med Chem., 1999, 42, 4172.

30. Pulkrábek J, Šroller J and Zahradníček J, Rostlinná Výroba, 1999, 45, 379.

31. Henselová M, Vizárová G and Macháčková I, Rostlinná Výroba, 2001, 47, 411.

32. Foltínová P, Sutoris V and Blockinger G, Folia Microbiologica, 1978, 23, 225.

33. Grandolini G, Ambrogi V and Rossi C, J Med Chem., 1986, 21, 455.

34. Afsah S A and Nayer S A, Asian J Chem., 1996, 8, 419.

35. Krieg M and Billitz J, Molecular and Biochemical Pharmacology, 1996,51,1461.

36. Sharma T C, Pawar S R and Reddy N J, Acta Chim Hung., 1983, 112, 159. 


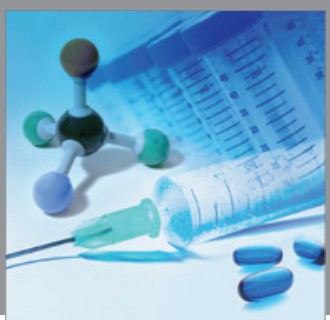

International Journal of

Medicinal Chemistry

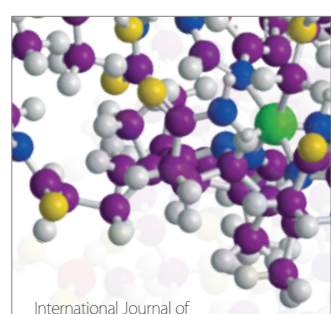

Carbohydrate Chemistry

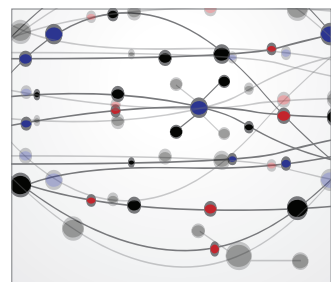

The Scientific World Journal
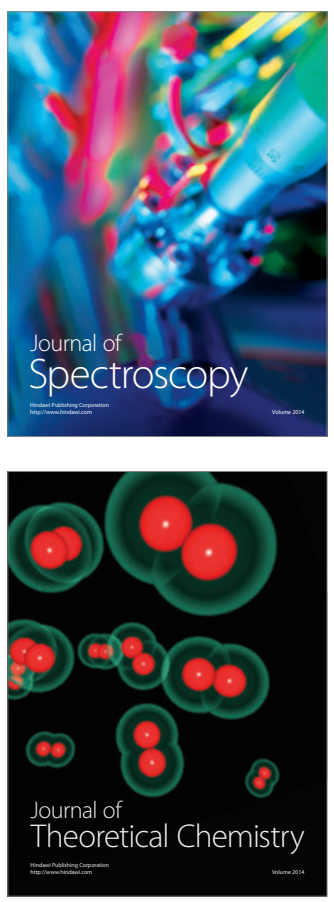
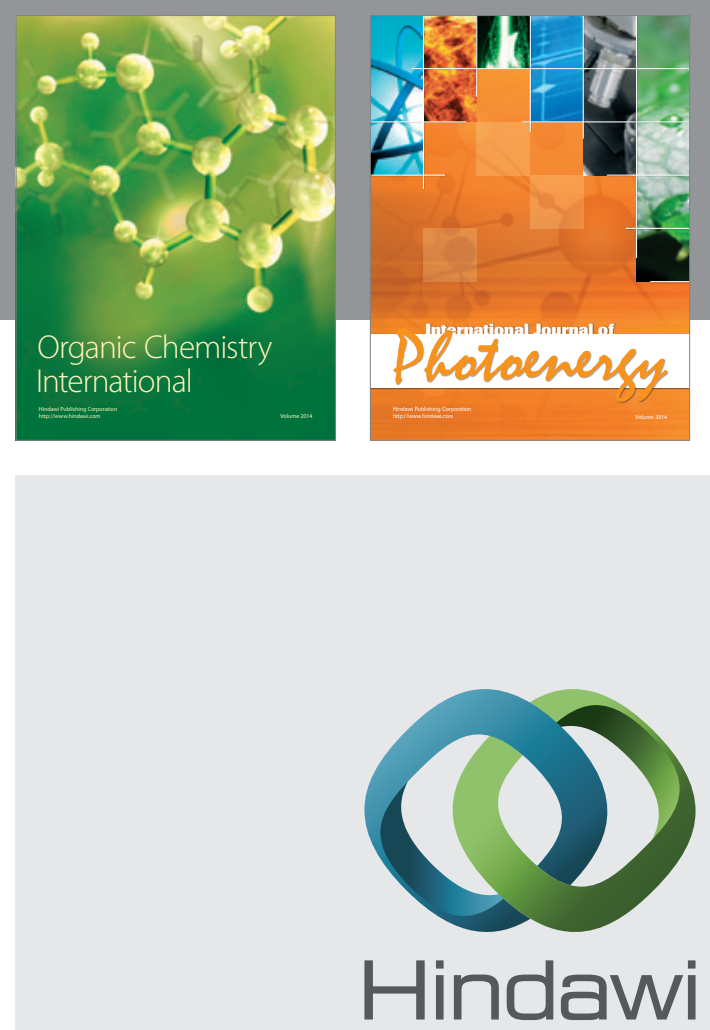

Submit your manuscripts at

http://www.hindawi.com
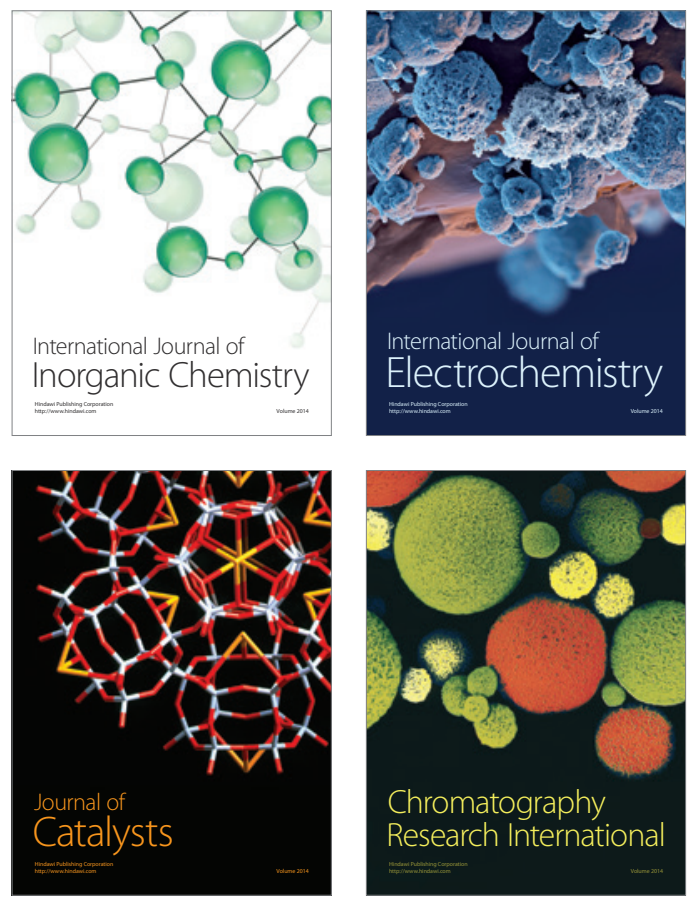
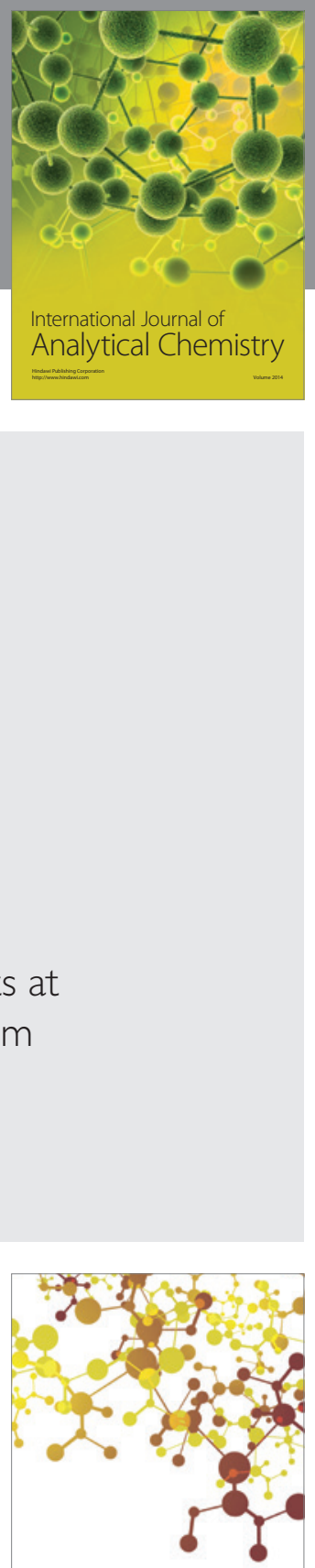

Journal of

Applied Chemistry
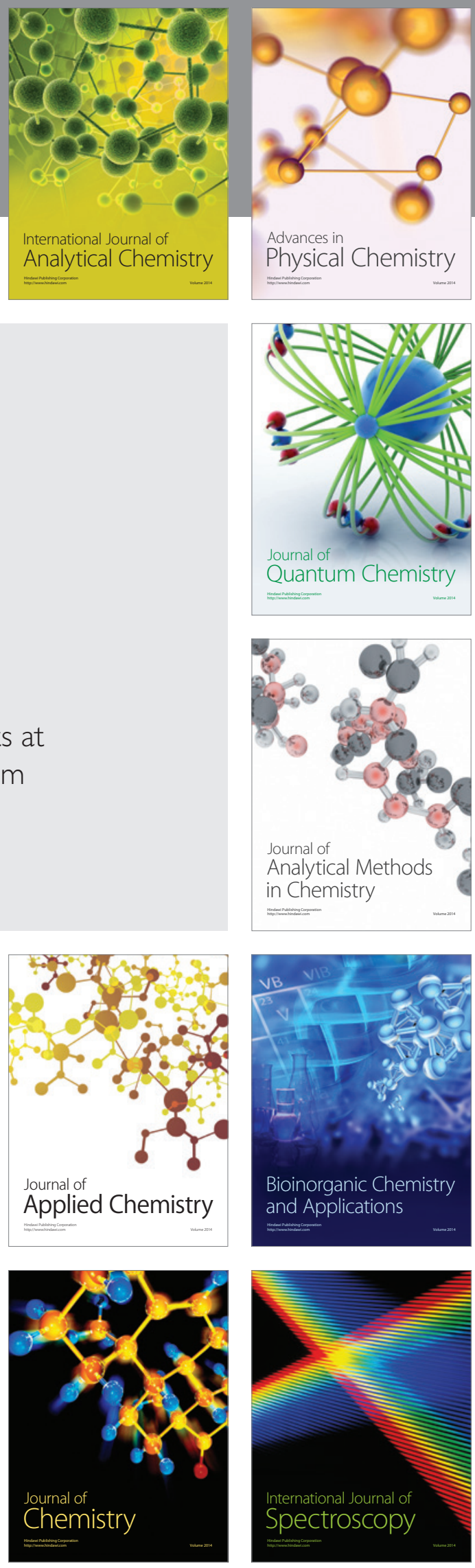